\title{
Knockdown of CFTR enhances sensitivity of prostate cancer cells to cisplatin via inhibition of autophagy
}

\author{
Q. ZHU*, H. LI, Y. LIU, L. JIANG \\ ${ }^{1 s t}$ Department of Urinary Surgery, Cangzhou Central Hospital, Hebei, 061000, People’s Republic of China \\ ${ }^{*}$ Correspondence: zhuqh_czch@sina.com
}

Received November 18, 2016 / Accepted February 25, 2017

\begin{abstract}
Prostate cancer is one of the most lethal diseases in men worldwide. Although the survival rate of men diagnosed with prostate cancer has increased with the improvement of treatments, drug resistance still remains a big challenge for improving overall survival. Cystic fibrosis transmembrane conductance regulator (CFTR), a cAMP-activated anion channel, has been reported to have a pivotal role in the pathogenesis of various cancers, but its role in chemoresistance of prostate cancer cells is poorly understood. In our study, we found that CFTR expression was significantly increased in prostate cancer tissues associated the chemoresistance, and in the cisplatin-resistant cell line LNCaP/CP compared with their respective parental cells. Cisplatin treatment inhibited CFTR expression in a concentration-dependent manner, which was correlated with a decrease in cell viability. Moreover, inhibition of CFTR by transfection of small interfering RNA enhanced cisplatin-induced the decrease of cell viability. Autophagy was dramatically increased in LNCaP/CP cells, as evidenced by autopaphgic markers as well as fluorescence microscopy analysis of GFP-LC3, MDC and AO staining. Of note, inhibiting autophagy by 3MA induced LNCaP/CP cell apoptosis, showed by MTT assay and Hoechst 33258 staining. In addition, blockade of CFTR also inhibited LNCaP/CP cell viability and autophagy. Furthermore, the dephosphorylation of AKT and mTOR was reversed by CFTR inhibition, indicating the knockdown of CFTR might inhibit autophagy in LNCaP/CP cells via activation of AKT/ mTOR signaling. Altogether, these results provide a novel understanding of the mechanism for acquired cisplatin. Inhibition of CFTR may be a useful strategy to increase the efficacy of cisplatin to treat prostate cancer by preventing the protective response of autophagy.
\end{abstract}

Key words: cisplatin, chemoresistance, prostate cancer, autophagy, CFTR

Prostate cancer is the most common malignant cancer and the 5th leading cause of cancer-related death in males [1]. Patients with prostate cancer are currently received surgery, radiotherapy, hormone therapy, biotherapy and chemotherapy treatments [2]. Notably, chemotherapy is a major therapy for prostate cancer, especially for advanced prostate cancer [3]. However, the poor response to chemotherapy is still a major critical prognostic factor in prostate cancer patients because of the progressive development of drug resistance. The molecular basis of acquired chemoresistance in prostate cancer is poorly understood and continues to be a major complication for chemotherapy [4]. Thus, it is of importance to understand the molecular mechanisms of chemoresistance and improve the clinical outcome of prostate cancer patients.

Although previous studies have suggested various mechanisms of chemoresistance, autophagy plays a critical role in the develop- ment of chemoresistance [5-7]. Autophagy is an evolutionarily conserved catabolic process that could degrade unnecessary or dysfunctional cytoplasmic components via endosomes and lysosomes to maintain the cellular energy supply and homeostasis $[8,9]$. Accumulating evidences have unveiled that autophagy is not only related to cell growth, differentiation and apoptosis, but also associated with the development of various diseases, such as metabolic diseases, infectious diseases and cancers [9, 10]. More importantly, it has been reported that autophagy acts as a protective mechanism to promote cancer cell survival and facilitate their chemoresistance towards the antineoplastic therapies such as cisplatin, doxorubicin and many other drugs $[6,11,12]$. However, the mechanisms by which cancer cells trigger autophagy and subsequently cause chemoresistance are still unclear.

Cystic fibrosis transmembrane conductance regulator (CFTR) is a cAMP-activated anion channel expressed in a wide 
variety of tissues [13]. Mutations or dysregulation of CFTR will result in cystic fibrosis (CF), a common autosomalrecessivedisorder in Caucasian populations [14]. Notably, recently, multiple studies have demonstrated that aberrant expression or function of CFTR may be also associated with cancer incidence [15-17]. A large cohort studies showed a marked increase in the risk of digestive tract cancers among CF patients in the United States [16]. Additionally, another studies also revealed an elevated risk of malignancies for the kidney, thyroid lymphoma, skin and prostate in CF patients [17-19]. Consequently, the association between CFTR and cancer risk indicates that CFTR plays a pivotal role in cancer progression. However, reports addressing the biological role of CFTR in prostate cancer development and drug resistance are limited. In this study, we showed that the increased CFTR expression may be associated with cisplatin resistance in prostate cancer cells. Blockade of CFTR sensitizes prostate cancer cells to cisplatin treatment through inhibition of autophagy, suggesting a novel strategy to increase the efficacy of cisplatin to treat prostate cancer.

\section{Patients and methods}

Materials and reagents. Fetal bovine serum (FBS), penicillin and streptomycin were purchased from Gibco (Grand Island, NY, USA). Cisplatin, acridine orange (AO), monodansylcadaverine (MDC), 3-methyladenine (3MA), 3-3' diaminobenzidine tetrahydrochloride (DAB) and Hoechst 33258 were obtained from Sigma Aldrich (St. Louis, MO, USA).

Patients and specimen preparation. In this study, the specimens of tumor tissue were obtained from 40 patients (20 with chemo-sensitive and 20 with chemo-resistant prostate cancer), who had been received histopathologic examination and surgical resection at Shanghai Shuguang Hospital. All experimental protocols were approved by Shanghai Shuguang Hospital and written informed consent was obtained from all patients whose specimens were used for this study.

Cell culture. Human prostate cancer cell line LNCaP was obtained from American Type Culture Collection (ATCC, Rockville, MD, USA) and cultured in RPMI1640 containing $10 \% \mathrm{FBS}, 100 \mathrm{U} / \mathrm{ml}$ penicillin and $100 \mu \mathrm{g} / \mathrm{ml}$ streptomycin in a humidified atmosphere of $5 \% \mathrm{CO}_{2}$ at $37^{\circ} \mathrm{C}$. The cisplatinresistant $\mathrm{LNCaP}(\mathrm{LNCaP} / \mathrm{CP})$ cells were developed from the parental LNCaP cells using an intermittent stepwise selection protocol as previously described [20,21].

Immunohistochemistry. The prostate cancer tissues were fixed in $10 \%$ formaldehyde, and embedded in paraffin. Paraffin sections were cut at $5 \mu \mathrm{m}$ and deparaffinized in xylene three times for 10 minutes each, and then transferred through a series of concentrations of ethanol for rehydration. After antigen retrieval, the sections were immersed in $1 \% \mathrm{H}_{2} \mathrm{O}_{2}$ for $10 \mathrm{~min}$ and incubated with the CFTR antibody (dilution 1:50, Santa Cruz Biotechnology, Inc., Paso Robles, CA, USA) at $4{ }^{\circ} \mathrm{C}$ overnight. The sections were incubated with the biotin- labeled anti-rabbit antibody (Santa Cruz Biotechnology, Inc.) for $1 \mathrm{~h}$ at room temperature. $\mathrm{DAB}$ was used as the substrate for the final visualization. The sections were counterstained with hematoxylin and then observed under a light microscope (Olympus, Tokyo, Japan). The intensity of CFTR was visually graded by a board-certified pathologist as $0-2$ (negative staining), 3-5 (weak staining), 6-8 (moderate staining) and 9-12 (strong staining). A total of 40 cases (chemo-sensitive and chemo-resistant tissues) were scored.

Western blot analysis. Cells were extracted with RIPA lysis buffer (Beyotime, Jiangsu, China) containing protease and phosphatase inhibitors (Roche Applied Science, Indianapolis, IN, USA). The protein content was determined by Protein Assay Kit (Beyotime). Equal amount of protein were separated by $8 \%$ SDS-PAGE and then transferred to polyvinylidene fluoride membranes (Bio-Rad, Hercules, CA, USA). The membranes were blocked with $5 \%$ non-fat milk and then incubated with the following primary antibodies: CFTR (dilution 1:500), Beclin-1, Atg5 and GAPDH (dilution 1:1000) (Santa Cruz Biotechnology, Inc.); LC-3, p62 (dilution 1:500), p-mTOR, mTOR, p-AKT and AKT (dilution 1:1000) (Cell Signaling Technology, Danvers, MA, USA). After washing with PBS 3 times, the membranes were further probed with appropriate secondary antibodies (peroxidase-conjugated anti-mouse or rabbit antibody, dilution 1:1000, Cell Signaling Technology). The signals were visualized by enhanced chemiluminescence (GE Healthcare, Princeton, NJ, USA). The relative band intensity was quantified using the ImageJ software (NIH, Bethesda, Maryland, USA).

Quantitative polymerase chain reaction (qPCR). Total RNA from LNCaP or LNCaP/CP cells was isolated using RNAeasy kit according to the manufacturer's instructions (Qiagen, Valencia, CA, USA). cDNA was synthesized from $1 \mu \mathrm{g}$ RNA using High Capacity cDNA Archive Kit (Applied Biosystems, Carlsbad, CA, USA). qPCR was performed using the following primers and SYBR Premix Ex Taq (Takara Bio, Inc., Ostu, Japan) in an Applied Biosystems 7500 RealTime PCR System. The primers used for amplification are as follows: CFTR sense TAGGAGCTTGAGCCCAGACG and antisense AACATCGCCGAAGGGCATTA; 18sRNA sense TCCTCTAAATGACCAAGTTTG and antisense GGAAGGGRTGTATTTATTAG. All primer sets were run under the following cycling conditions: $95^{\circ} \mathrm{C}$ for $30 \mathrm{sec}$ followed by 40 cycles of denaturation $\left(95^{\circ} \mathrm{C}\right.$ for $30 \mathrm{sec}, 60^{\circ} \mathrm{C}$ for $45 \mathrm{sec}$ and $72^{\circ} \mathrm{C}$ for $30 \mathrm{sec}$ and annealing and extension $\left(72^{\circ} \mathrm{C}\right.$ for $\left.1 \mathrm{~min}\right)$. The expression for each gene was calculated using the $2^{-\Delta \Delta \mathrm{CT}}$ method and 18srRNA was used as an internal control.

Cell viability assay. Cell viability was determined by 3-(4,5-dimethylthiazol-2-yl)-2,5-diphenyltetrazolium bromide (MTT) assay as previously described [22]. Briefly, LNCaP or LNCaP/CP cells were seeded in 96-well plates at a density of 10,000 cells per well. After corresponding treatments, cells were incubated with MTT at final concentration of $500 \mu \mathrm{g} / \mathrm{ml}$ for $1 \mathrm{~h}$. Subsequently, the medium was removed and formazan formed was dissolved in dimethyl sulfoxide (DMSO). The ab- 
sorbance was read with a microplate reader (SpectraMax M5 Molecular Device, Sunnyvale, CA, USA) at $540 \mathrm{~nm}$.

Transfection of small interfering RNA (siRNA). All reagents for gene silencing were obtained from Qiagen. The target sequence of the siRNA against human CFTR gene was 5'TCGATATATTACTGTCCACAA3'. Cells were transfected with siRNA for CFTR or a negative siRNA (NS) using Hiperfect reagent according to the manufacture's instructions. After $6 \mathrm{~h}$, the cells were replaced with compete medium for $48 \mathrm{~h}$.

GFP-LC3 transient transfection. Cells were transiently transfected with GFP-LC3 vector (a kind gift from Dr. Runtian Liu, the Second Hospital of Hebei Medical University, Hebei, China) using Lipofectamine 2000 (Invitrogen, Carlsbad, CA, USA) according to the manufacturer's guidelines. After $24 \mathrm{~h}$, GFP immunofluorescence was observed by a confocal microscopy (LSM 5 Pascal Microscope, Carl Zeiss, Oberkochen, Germany).

AO staining. $\mathrm{LNCaP}$ or $\mathrm{LNCaP} / \mathrm{CP}$ cells were seeded in 6-well plates overnight. After washed with $\mathrm{PBS}$ three times, cells were incubated with $2 \mathrm{mg} / \mathrm{ml}$ of $\mathrm{AO}$ for $15 \mathrm{~min}$ at $37^{\circ} \mathrm{C}$. Cell were rinsed by PBS three times and immediately inspected under a confocal microscopy (excitation, $488 \mathrm{~nm}$; emission, $640 \mathrm{~nm}$ ) (LSM 5 Pascal Microscope, Carl Zeiss).

MDC staining. LNCaP or LNCaP/CP cells were cultured in 6-well plates and permited to attach by overnight incubation. Cells were washed three times with PBS and incubated with 1 $\mathrm{mg} / \mathrm{ml}$ of $\mathrm{MDC}$ for $30 \mathrm{~min}$ at $37^{\circ} \mathrm{C}$. Autophagic vacuoles with MDC staining were observed under by a confocal microscopy (excitation, $390 \mathrm{~nm}$; emission, $460 \mathrm{~nm}$ ) (LSM 5 Pascal Microscope, Carl Zeiss).

Hoechst 33258 staining. The apoptotic morphology of LNCaP and LNCaP/CP cells was examined by Hoechst 33258 staining. After corresponding treatments, cells were fixed in $4 \%$ paraformaldehyde for $1 \mathrm{~h}$ at room temperature and then were incubated with $10 \mu \mathrm{g} / \mathrm{ml}$ of Hoechst 33258 for $10 \mathrm{~min}$. The morphological changes were observed under a confocal microscopy (LSM 5 Pascal Microscope, Carl Zeiss) and the percentage of apoptotic cells was quantified with ImageJ software.

Statistical analysis. All data were presented as mean \pm SEM. The regression analysis was determined by the Pearson correlation test. The Wilcoxon-Mann-Whitney rank sum test was used to calculate the statistical significance of immunohistochemistry score between chemo-sensitive and chemo-resistant tumor tissues. The significance between groups was determined by one-way analysis of variance (ANOVA) or the unpaired two-tailed Student's t test using SPSS 17.0 statistical software (SPSS Inc., Chicago, IL, USA). P value less than 0.05 was considered to be statistically significant.

\section{Results}

CFTR expression is correlated with cisplatin-resistance in prostate cancer. Figure $1 \mathrm{~A}$ showed representative photos of CFTR expression of chemo-sensitive and chemo-resistant prostate cancer tissues by immunohistochemistry staining. As shown in Figure 1B, the overall staining of CFTR was increased in chemo-resistant prostate cancer tissues, as compared with chemo-sensitive prostate cancer tissues. In accord with the results from human tissue samples, higher expression of CFTR was also found in cisplatin-resistant $\mathrm{LNCaP} / \mathrm{CP}$ cells than in parent LNCaP cells (Figure 1C). Similarly, expression level of CFTR was further confirmed by qPCR assay (Figure 1D), indicating the changes of CFTR expression may be associated the cisplatin resistance in prostate cancer. Thus, we next investigate the effect of cisplatin on CFTR expression. Western blot results showed that CFTR protein expression was decreased in a concentration-dependent manner after cisplatin treatment for $24 \mathrm{~h}$ (Figure 1E). Moreover, cisplatin also decreased LNCaP cell viability in a concentrationdependent manner. Compare with control group, at 5, 10, 20, $40 \mu \mathrm{M}$ of cisplatin, cell viability was reduced to $89.2 \pm 8.7 \%$, $78.5 \pm 8.3 \%, 68.7 \pm 7.4 \%$ and $54.1 \pm 6.4 \%$, respectively (Figure $1 F)$. Interestingly, the regression analysis showed that CFTR protein expression was positively correlated with the cell viability with the correlation coefficient of 0.868 (Figure 1G), further indicating CFTR expression may play an important role in the development of cisplatin-resistant prostate cancer. We tested this by knocking down CFTR expression in LNCaP cells. CFTR siRNA at $10 \mathrm{nM}$ significantly decreased endogenous CFTR expression more than $70 \%$. Moreover, no significant differences were observed between $10 \mathrm{nM}$ and $20 \mathrm{nM}$ (Figure $1 \mathrm{H}$ ). Therefore, we chose CFTR at $10 \mathrm{nM}$ for $48 \mathrm{~h}$ in the following experiments. As displayed in Figure 1I, CFTR expression was further decreased after cisplatin treatment in cells transfected with CFTR siRNA. MTT assay showed that CFTR knockdown had no effects on cell viability in LNCaP cells (data not shown). However, inhibition of CFTR further enhanced cisplatin-induced the decrease of LNCaP cell viability (Figure 1J).

Autophagy is induced in cisplatin-resistant prostate cancer cells. To explore the role of autophagy in the development of cisplatin-resistant prostate cancer, autophagic evidence and markers were examined in $\mathrm{LNCaP}$ and $\mathrm{LNCaP} / \mathrm{CP}$ cells. MDC staining (autophagic vacuoles) and AO staining (acidic vesicular organelles) showed that autolysosomes were highly accumulated in LNCaP/CP cells (Figure 2A and B). Moreover, we observed GFP-LC3 punctate dots distribution as a marker for autophagosome formation in human prostate cancer cells. As shown in Figure 2C, GFP-LC3 transfected LNCaP/CP cells also showed a significant accumulation of GFP-LC3 puncta when compared with LNCaP cells. To further corroborate the results in the fluorescence microscopy analysis, protein expression of LC3, Beclin-1, Atg-5 and p62 were determined by western blot. The conversion LC3B-I to LC3B-II was enhanced in LNCaP/CP cells, which implies an increase of autophagosomes within cells (Figure 2D). Similarly, the protein expression of another two autophagic markers, Beclin-1 (also known as Atg6) and Atg5 were also increased in $\mathrm{LNCaP} / \mathrm{CP}$ cells, as compared with LNCaP cells (Figure $2 \mathrm{E}$ 
A

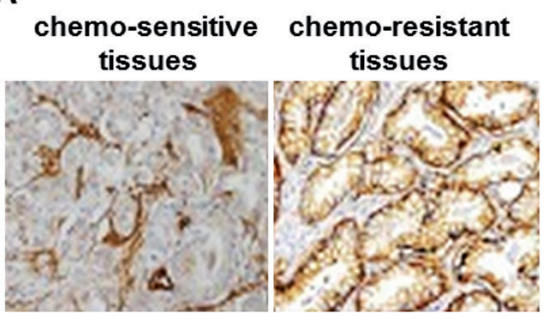

E

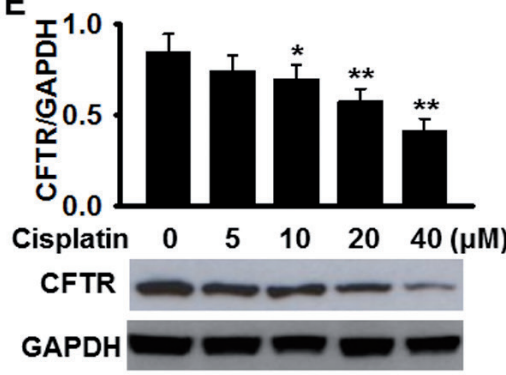

H

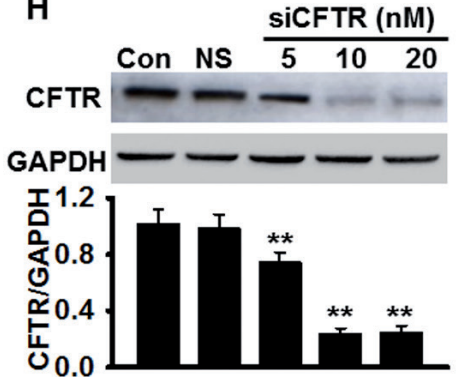

B

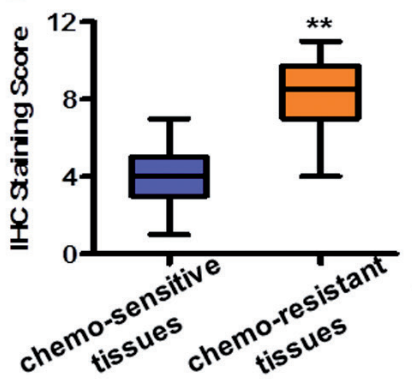

$F$

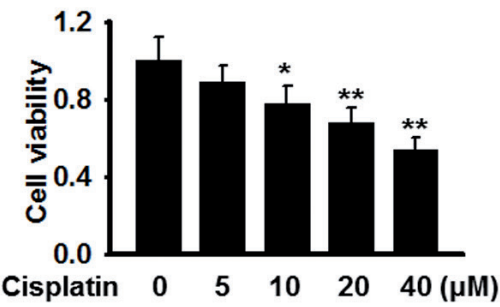

I

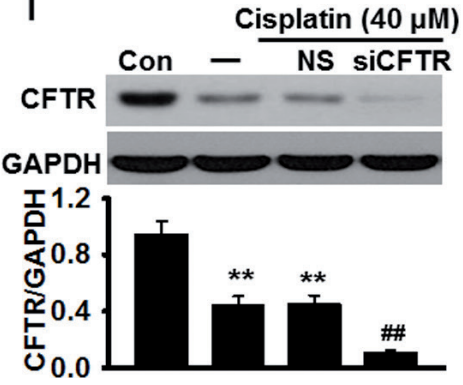

D
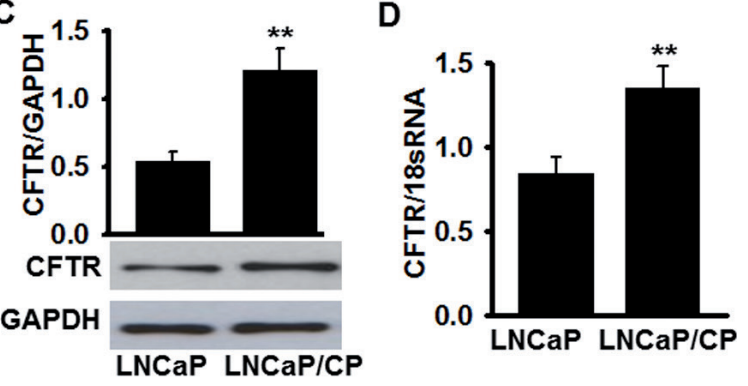

G

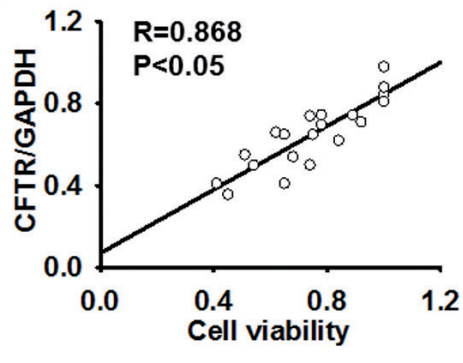

J

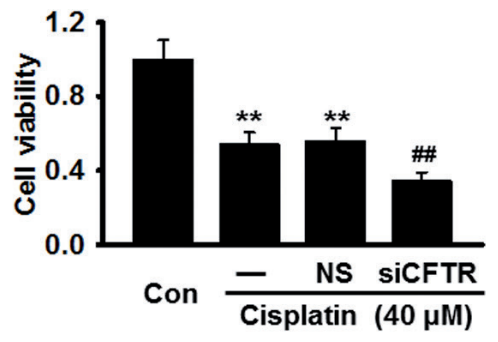

Figure 1. CFTR expression is associated with cisplatin resistance in prostate cancer cells. (A) CFTR expression in chemo-sensitive or chemo-resistant prostate cancer tissues was determined immunohistochemistry. Representative images were shown. (B) A box-plot was generated for CFTR immunohistochemistry (IHC) staining score between chemo-sensitive and chemo-resistant tumor tissues. ${ }^{* *} \mathbf{P}<0.01$ vs. chemo-sensitive prostate cancer tissues, $\mathbf{n}=\mathbf{2 0}$ in each group. (C and D) CFTR expression in cisplatin-resistant LNCaP/CP cells and parent LNCaP cells was examined by western blot (C) and qPCR (D). ${ }^{* *} \mathbf{P}<0.01$ vs. LNCaP cells, $n=6$. (E) LNCaP cells were incubated with cisplatin at different concentration $(5,10,20$ and $40 \mu M)$ for 24 h. Western blot assay was used to analyze the effect of cisplatin on CFTR protein expression. (F) Cell viability was assessed by MTT assay. (G) Correlation between cell viability and CFTR expression was analyzed. (H) LNCaP cells were transfected with negative siRNA (NS) or CFTR siRNA (siCFTR) for 48 h, the inference effect was assessed by western blot. (I) LNCaP cells were transfected with NS or siCFTR (10 nM) for $48 \mathrm{~h}$, followed by incubation of cisplatin (40 $\mu \mathrm{M}$ ) for another $24 \mathrm{~h}$. CFTR expression were examined by western blotting. (J) Cell viability was analyzed by $\mathrm{MTT}$ assay. ${ }^{\star} \mathrm{P}<0.05$, ${ }^{\star \star} \mathrm{P}<0.01$ vs. control; $\# \mathbf{P}<0.01$ vs. cisplatin alone, $\mathrm{n}=6$.

and F). However, the expression of p62, which accumulated in cells with reduced autophagy [23], was remarkably lower in LNCaP/CP cells than in LNCaP cells (Figure 2G).

Inhibition of autophagy induces apoptosis in cisplatin-resistant prostate cancer cells. Autophagy has been suggested to serve as a pro-survival mechanism that facilitates cancer cells to resistance therapy-induced cell death [7]. To verify this consumption, we firstly analyzed cell viability in LNCaP cells or LNCaP/CP cells in the presence or absence of 3MA. MTT assay showed that 3MA alone failed to affect cell viability in LNCaP cells, which 3MA dramatically inhibited the increase of cell viability in LNCaP/CP cells (Figure 3A). Furthermore, Hoechst 33258 staining was used to examine the apoptosis of cells with or without 3MA. Consistently, 3MA also produced no effects on apoptosis in LNCaP cells. However, the number of apoptotic LNCaP/CP cells, was much more in the group with $3 \mathrm{MA}$ treatment than in the group without (Figure 3B and C), suggesting that autophagy contributes to, at least partially, the cisplatin resistance in prostate cancer cells. In addition, to explore the possibility whether inhibition of autophagy re-sensitizes LNCaP/CP to cisplatin treatment, $\mathrm{LNCaP} / \mathrm{CP}$ cells were co-incubated with $3 \mathrm{MA}$ and cisplatin for $24 \mathrm{~h}$. The data showed that $\mathrm{LNCaP} / \mathrm{CP}$ cell viability was slightly decreased after cisplatin treatment. Importantly, inhibition of autophagy using 3MA resulted in a more pronounced decrease of cell viability in $\mathrm{LNCaP} / \mathrm{CP}$ 

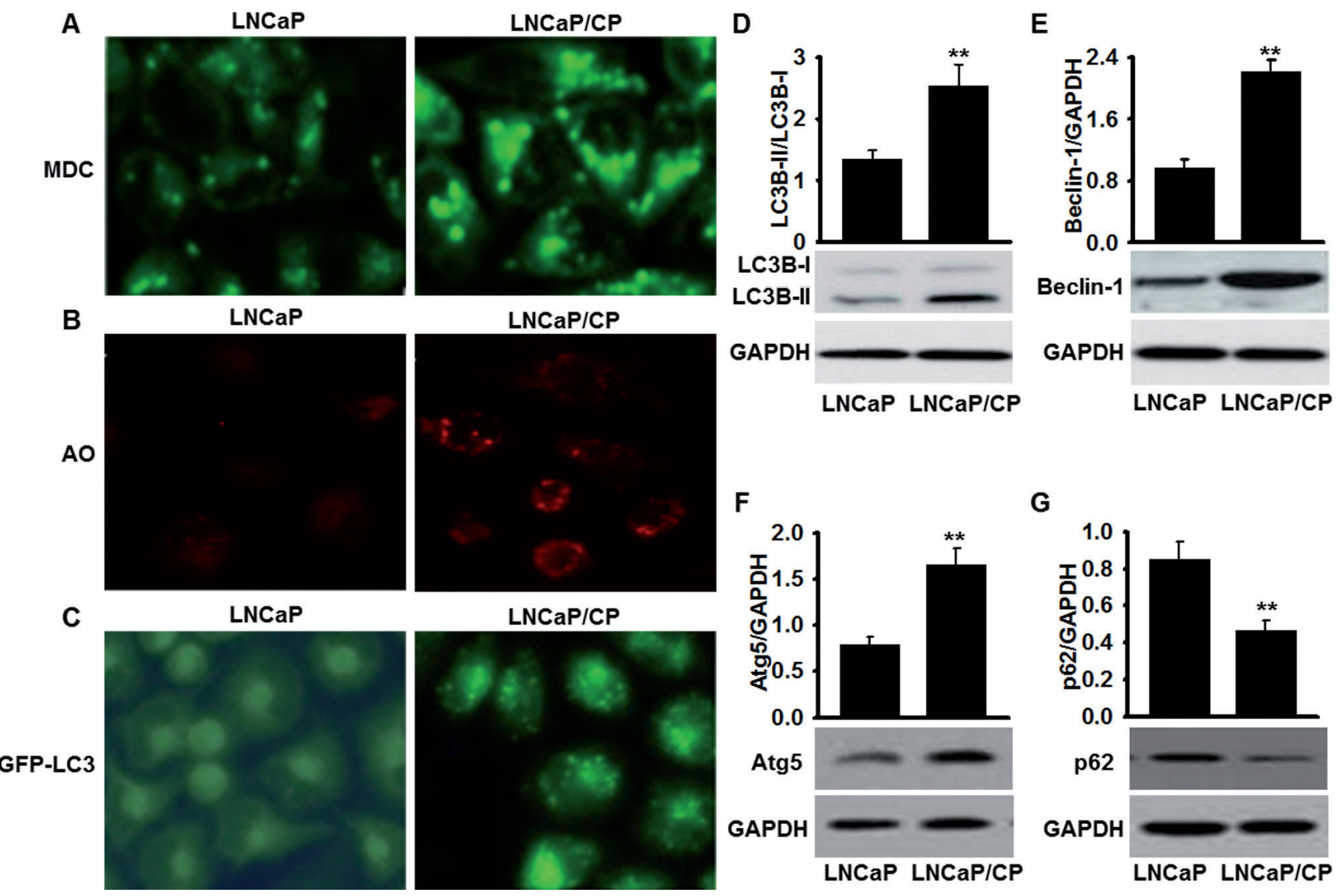

Figure 2. Induction of autophagy cisplatin-resistant prostate cancer cells. (A) Autophagic vacuoles were detected by MDC staining. (B) acidic vesicular organelles were observed by AO staining. (C) LNCaP cells or LNCaP/CP cells were transfected with GFP-LC3 vector for $24 \mathrm{~h}$ and the formation of GFPLC3 puncta was observed under a confocal microscopy. (D-G) The protein expression of LC3B (D), Beclin-1 (E), Atg5 (F) and p62 (G) were determined by western blot. ${ }^{* *} \mathrm{P}<0.01$ vs. $\mathrm{LNCaP}$ cells, $\mathrm{n}=6$.

cells treated with cisplatin (Figure 3D). Overall, these results indicate that cisplatin causes chemoresistance in $\mathrm{LNCaP} / \mathrm{CP}$ via induction of autophagy.

Blockade of CFTR decreases cisplatin resistance via suppression of autophagy in prostate cancer cells. Our results showed that blockade of CFTR could promote cisplatin-induced the decrease of LNCaP cell viability, while inhibition of autophagy caused apoptosis in LNCaP/CP cells. Therefore, we investigated whether blockade of CFTR can attenuate cisplatin resistance via inhibition of autophagy induced by cisplatin. Firstly, we analyzed the effects of CFTR inhibition on cell viability in LNCaP cells and LNCaP/CP cells. As shown in Figure 4A, knockdown of CFTR had no effect on LNCaP cell viability, while LNCaP/CP cell viability was significantly inhibited after CFTR inhibition. Moreover, blockade of CFTR reversed cisplatin resistance-induced the accumulation of GFP-LC3 puncta in LNCaP/CP cells (Figure $4 \mathrm{~B}$ ). In addition, the increase of the ratio of LC3B-II to LC3B-I and Beclin-1 in LNCaP/CP cells were abrogated after CFTR inhibition. Consistently, blockade of CFTR produced no effects on the accumulation of GFP-LC3 puncta, the ratio of LC3B-II to LC3B-I, and Beclin-1 expression in LNCaP cells (Figure 4C and D).

Knockdown of CFTR inhibited cisplatin resistanceinduced autophagy through activation of AKT/mTOR signaling. AKT/mTOR signaling plays a vital role in negatively regulating autophagic activity [22]. To investigate the mechanism by which CFTR inhibition suppresses autophagy in cisplatin-resistant LNCaP cells, components of AKT/ mTOR signaling pathway were examined by western blot analysis. As shown in Figure 5A, blockade of CFTR did not change the phosphorylation of AKT in LNCaP cells. Interestingly, we found that AKT phosphorylation was markedly downregulated in $\mathrm{LNCaP} / \mathrm{CP}$ cells, which was reversed by CFTR inhibition. Consistent with the restoration of AKT, the phosphorylation of mTOR, an important downstream kinase of AKT, was also increased in LNCaP/CP cells (Figure 5B). These data has confirmed the involvement of AKT/mTOR signaling in CFTR-mediated autophagy in cisplatin resistant prostate cancer cells. 


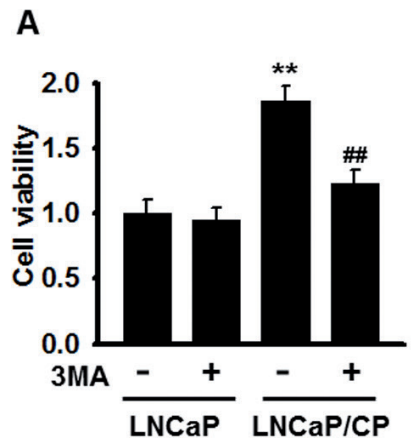

B

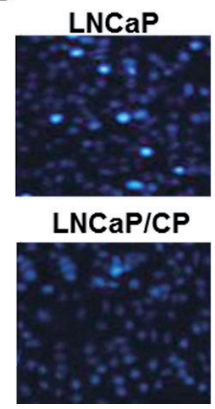

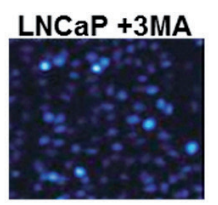

LNCaP/CP+3MA

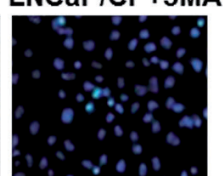

C

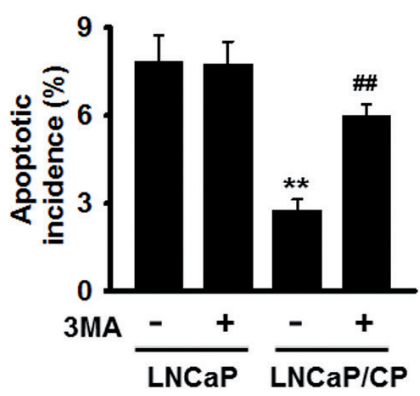

D

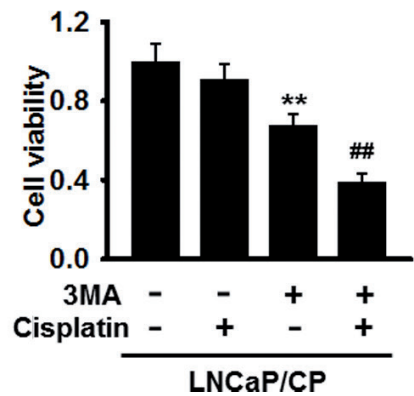

Figure 3. Blockade of autophagy causes apoptosis in cisplatin-resistant prostate cancer cells. (A) Cisplatin-resistant LNCaP/CP cells or parent LNCaP cells were treated with or without $3 \mathrm{MA}(5 \mathrm{mM})$ for $24 \mathrm{~h}$, cell viability was determined by MTT assay. (B) Apoptosis in LNCaP cells and LNCaP/CP cells was analyzed by the use of Hoechst 33258 staining. (C) Apoptotic incidence was quantified with ImageJ software. ${ }^{\star \star} \mathrm{P}<0.01 \mathrm{vs}$. LNCaP cells; \#\#P<0.01 vs. $\mathrm{LNCaP} / \mathrm{CP}$ cells alone, $\mathrm{n}=6$. (D) $\mathrm{LNCaP} / \mathrm{CP}$ cells were co-incubated with $3 \mathrm{MA}$ and cisplatin for $24 \mathrm{~h}$, cell viability was determined by MTT assay. ${ }^{* *} \mathrm{P}<0.01$ vs. control; \#\#P<0.01 vs. cisplatin treatment alone, $\mathrm{n}=4$.

\section{Discussion}

Among many anti-cancer chemotherapeutic agents, cisplatin is one of the most chemotherapy which is used to treat many solid tumors including breast cancer, prostate cancer and non-small cell lung cancer, etc. [3, 24, 25]. However, drug resistance is increasing in the development of prostate cancer after cisplatin treatment [4]. Despite numerous studies are
A

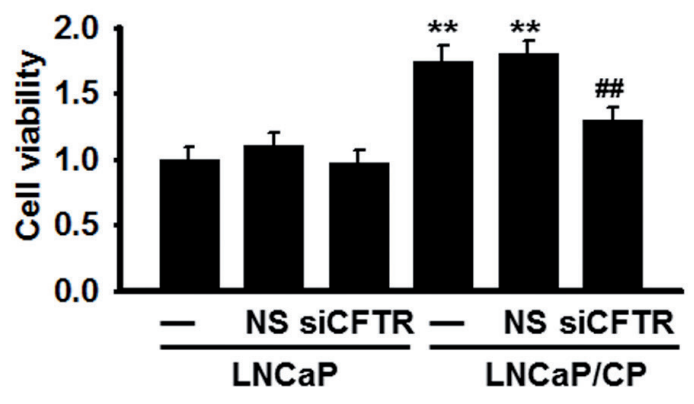

C

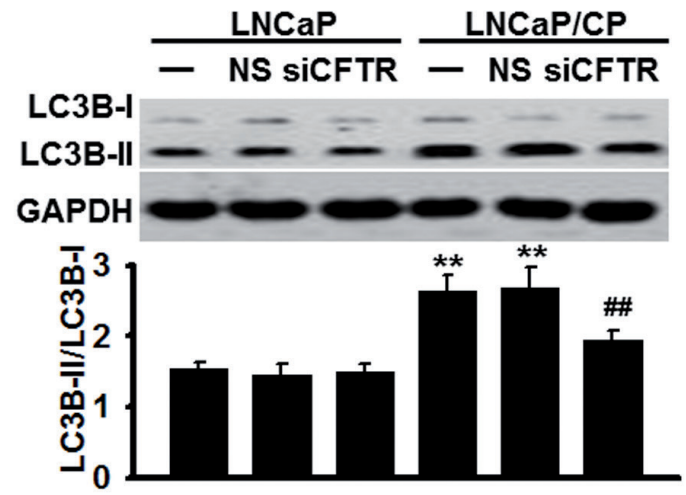

LNCaP

B
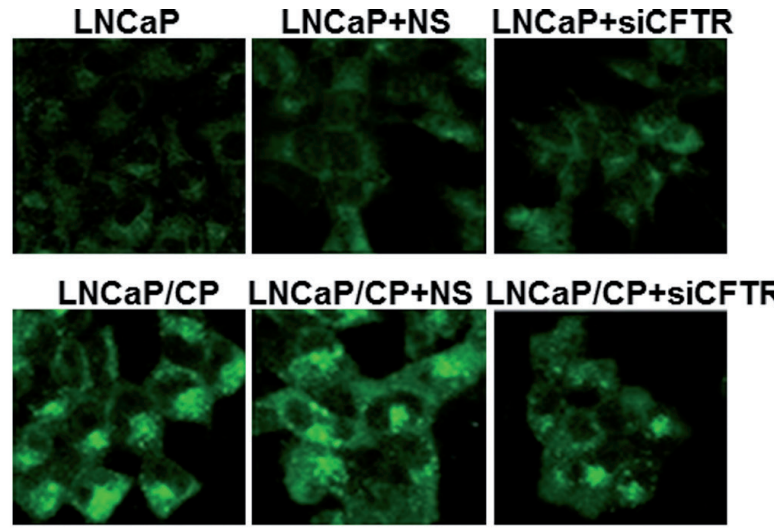

D

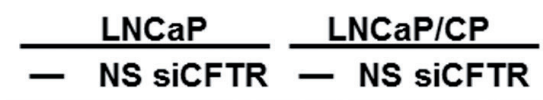

Beclin-1

GAPDH

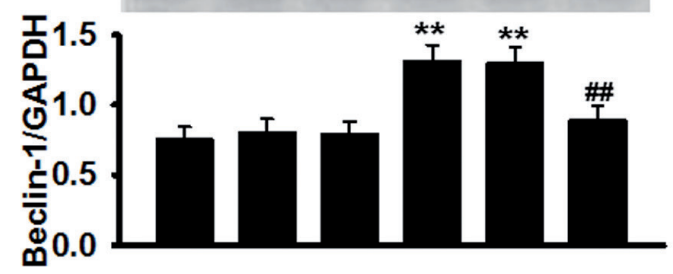

Figure 4. CFTR decreases cisplatin resistance via inhibition of autophagy. (A) Cisplatin-resistant LNCaP/CP cells or parent LNCaP cells were transfected with negative siRNA (NS) or CFTR siRNA (siCFTR) $(10 \mathrm{nM})$ for $48 \mathrm{~h}$,cell viability was determined by MTT assay. (B) After the treatment mentioned in (A), cells were transfected with GFP-LC3 vector for $24 \mathrm{~h}$ and the formation of GFP-LC3 puncta was observed under a confocal microscopy. (C and D) The protein expression of LC3B (C) and Beclin-1 (D) were determined by western blot. ${ }^{*} \mathrm{P}<0.01$ vs. $\mathrm{LNCaP}$ cells; \#\#P<0.01 vs. $\mathrm{LNCaP} / \mathrm{CP}$ cells alone, $\mathrm{n}=6$. 

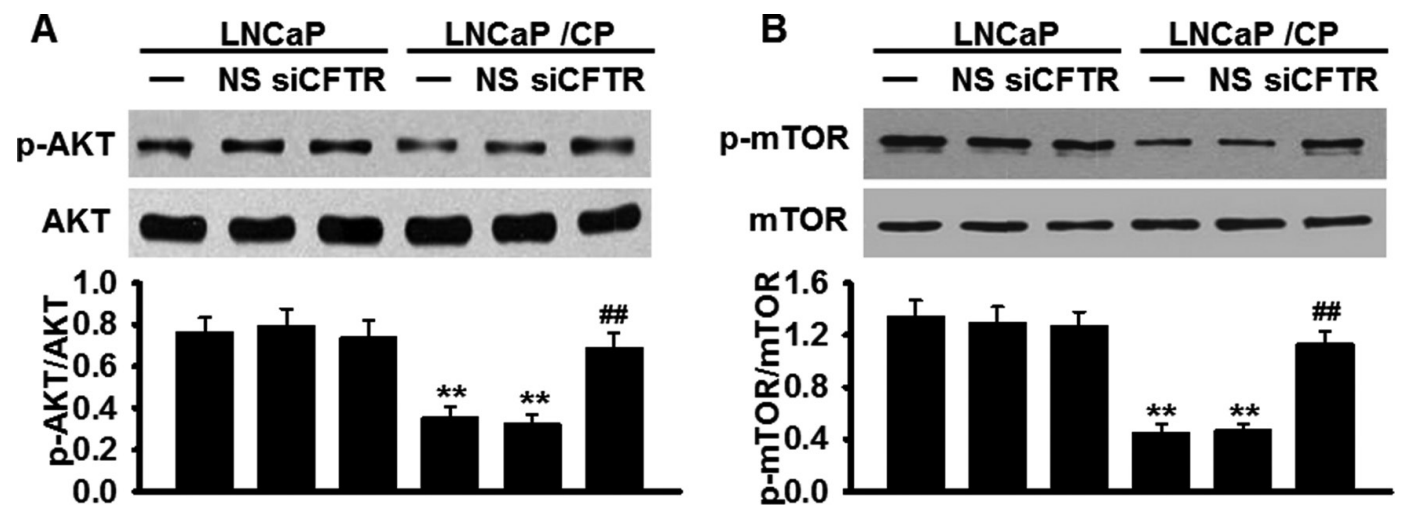

Figure 5. The involvement of AKT/mTOR signaling in CFTR-mediated autophagy in cisplatin resistant prostate cancer cells. (A and B) Cisplatin-resistant LNCaP/CP cells or parent LNCaP cells were transfected with negative siRNA (NS) or CFTR siRNA (siCFTR) (10 nM) for 48 h, the phosphorylation of AKT (A) and mTOR (B) were determined by western blot analysis. ${ }^{*} \mathrm{P}<0.01$ vs. $\mathrm{LNCaP}$ cells; $\# \# \mathbf{P}<0.01$ vs. $\mathrm{LNCaP} / \mathrm{CP}$ cells alone, $\mathrm{n}=6$.

focused to identify an effective novel approach to overcome cisplatin resistance in prostate cancer cells, the underpinning molecular mechanism is still poorly understood. Our current study demonstrated for the first time that CFTR expression was increased in chemo-resistant prostate cancer tissues and cisplatin-resistant prostate cancer cells. Moreover, cisplatin treatment resulted in a decrease in CFTR expression and downregulation of CFTR further augmented the cytotoxic effects of cisplatin in human prostate cancer LNCaP cells, suggesting the increased CFTR expression may act as a survival mechanism to facilitate cisplatin resistance in prostate cancer cells.

In the present study, we found that autophagy was induced in cisplatin-resistant $\mathrm{LNCaP} / \mathrm{CP}$ cells but not in parent $\mathrm{LNCaP}$ cells. This occurs with discoveries that cisplatin treatment can also trigger autophagy in gastric cancer cells, cervical cancer cells and squamous cell carcinoma cells $[5,6,26]$. Of note, there is a crosstalk between autophagy and apoptosis, and the function of autophagy in cancer is controversial and intricate. Several previous studies have shown that autophagy can trigger autophagic death pathway, which is the predominant cell death mechanism in response certain chemotherapy $[27,28]$. On the other hand, many studies have proved that autophagy can also protect cells from apoptosis and various stress to counteract cell death after chemotherapy $[6,7,11$, 29]. Therefore, autophagy is considered as an important target in intervention and treatment of cancers [30], and autophagymodulating agents also have been identified as potential cancer therapeutic agents [31]. For example, many autophagy inhibitors such as hydroxychloroquine, chloroquine and amodiaquine, have been widely used for tumor therapy [32-34]. Here, we demonstrated that the function of cisplatin-induced autophagy is a protective mechanism, because inhibition autophagy could inhibit cell viability and induced apoptosis in cisplatin-resistant LNCaP/CP cells, and re-sensitize them to cisplatin treatment. Moreover, CFTR inhibition abolished the induction of autophagy in cisplatin-resistant LNCaP/CP cells. These alterations may provide possible explanation why blockade of CFTR can increase chemosensitivity of cisplatin in prostate cancer cells.

We next investigated the mechanisms how CFTR mediates cisplatin-induced autophagy. It is worth noting that AKT/ mTOR was an important regulator upstream autophagy [22]. Activation of AKT can result in phosphorylation of the serine/ threonine kinase mTOR which is a major negative regulator of autophagy [27]. Moreover, cisplatin has been found to induce protective autophagy through AKT/mTOR signaling pathway in glioma cells and fibrosarcoma cells [35]. In the line with our results in autophagy detection, we found that the phosphorylation of AKT and mTOR were decreased in cisplatin-resistant LNCaP/CP cells. However, blockade of CFTR reversed the dephosphorylation of AKT and mTOR. Collectively, our data indicate that blockade of CFTR attenuates cisplatin resistance by inhibiting autophagy in prostate cancer cells.

In summary, our findings showed that CFTR expression was increased in chemo-resistant prostate cancer tissues or cells, which might contribute to the resistance towards chemotherapy. Blockade of CFTR inhibited cell viability and autophagy via activation of AKT/mTOR signaling pathway in cisplatin-resistant prostate cancer cells, suggesting that CFTR knockdown increases chemosensitivity of cisplatin via, at least in part, the inhibition of autophagy. This study has uncovered a novel role of CFTR in reducing the chemoresistance acquired towards the cisplatin treatment and provided potential therapeutic strategies for the treatment of human prostate cancer.

\section{References}

[1] YUAN S, WANG L, CHEN X, FAN B, YUAN Q et al. Triptolide inhibits the migration and invasion of human prostate cancer cells via Caveolin-1/CD147/MMPs pathway. Biomed Pharmacother 2016; 84: 1776-1782. https://doi.org/10.1016/j. biopha.2016.10.104 
[2] CHEN CD, WELSBIE DS, TRAN C, BAEK SH, CHEN R et al. Molecular determinants of resistance to antiandrogen therapy. Nat Med 2004; 10: 33-39. https://doi.org/10.1038/nm972

[3] YAN B, GUO Q, NAN XX, WANG Z, YIN Z et al. Microribonucleic acid 29b inhibits cell proliferation and invasion and enhances cell apoptosis and chemotherapy effects of cisplatin via targeting of DNMT3b and AKT3 in prostate cancer. Onco Targets Ther 2015; 8: 557-565.

[4] WANG Y, NANGIA-MAKKER P, BALAN V, HOGAN V, RAZ A. Calpain activation through galectin-3 inhibition sensitizes prostate cancer cells to cisplatin treatment. Cell Death Dis 2010; 1: e101. https://doi.org/10.1038/cddis.2010.79

[5] ZHANG HQ, HE B, FANG N, LU S, LIAO YQ et al. Autophagy inhibition sensitizes cisplatin cytotoxicity in human gastric cancer cell line SGC7901. Asian Pac J Cancer Prev 2013; 14: 4685-4688. https://doi.org/10.7314/APJCP.2013.14.8.4685

[6] XU Y, YU H, QIN H, KANG J, YU C et al. Inhibition of autophagy enhances cisplatin cytotoxicity through endoplasmic reticulum stress in human cervical cancer cells. Cancer Lett 2012; 314: 232-243. https://doi.org/10.1016/j. canlet.2011.09.034

[7] JIANG L, XU L, XIE J, LI S, GUAN Y et al. Inhibition of autophagy overcomes glucocorticoid resistance in lymphoid malignant cells. Cancer Biol Ther 2015; 16: 466-476. https:// doi.org/10.1080/15384047.2015.1016658

[8] RAVIKUMAR B, SARKAR S, DAVIES JE, FUTTER M, GARCIA-ARENCIBIA $M$ et al. Regulation of mammalian autophagy in physiology and pathophysiology. Physiol Rev 2010; 90: 1383-1435. https://doi.org/10.1152/ physrev.00030.2009

[9] KLIONSKY DJ. Autophagy revisited: a conversation with Christian de Duve. Autophagy 2008; 4: 740-743. https://doi. org/10.4161/auto.6398

[10] ARROYO DS, GAVIGLIO EA, PERALTA RAMOS JM, BUSSI C, RODRIGUEZ-GALAN MC et al. Autophagy in inflammation, infection, neurodegeneration and cancer. Int Immunopharmacol 2014; 18: 55-65. https://doi.org/10.1016/j. intimp.2013.11.001

[11] WU W, LI W, ZHOU Y, ZHANG C. Inhibition of beclin1 affects the chemotherapeutic sensitivity of osteosarcoma. Int J Clin Exp Pathol 2014; 7: 7114-7122.

[12] FONG MY, JIN S, RANE M, SINGH RK, GUPTA R et al. Withaferin A synergizes the therapeutic effect of doxorubicin through ROS-mediated autophagy in ovarian cancer. PLoS One 2012; 7: e42265. https://doi.org/10.1371/journal. pone.0042265

[13] COLLINS FS. Cystic fibrosis: molecular biology and therapeutic implications. Science 1992; 256: 774-779. https://doi. org/10.1126/science. 1375392

[14] O'SULlivaN BP, FREEDMAN SD. Cystic fibrosis. Lancet 2009; 373: 1891-1904. https://doi.org/10.1016/S01406736(09)60327-5

[15] SON JW, KIM YJ, CHO HM, LEE SY, LEE SM et al. Promoter hypermethylation of the CFTR gene and clinical/ pathological features associated with non-small cell lung cancer. Respirology 2011; 16: 1203-1209. https://doi. org/10.1111/j.1440-1843.2011.01994.x
[16] MAISONNEUVE P, MARSHALL BC, KNAPP EA, LOWENFELS AB. Cancer risk in cystic fibrosis: a 20-year nationwide study from the United States. J Natl Cancer Inst 2013; 105: 122-129. https://doi.org/10.1093/jnci/djs481

[17] DING S, GONG BD, YU J, GU J, ZHANG HY et al. Methylation profile of the promoter CpG islands of 14 „drug-resistance" genes in hepatocellular carcinoma. World J Gastroenterol 2004; 10:3433-3440. https://doi.org/10.3748/ wjg.v10.i23.3433

[18] XIE C, JIANG XH, ZHANG JT, SUN TT, DONG JD et al. CFTR suppresses tumor progression through miR-193b targeting urokinase plasminogen activator (uPA) in prostate cancer. Oncogene 2013; 32: 2282-2291. https://doi. org/10.1038/onc.2012.251

[19] ZHANG JT, JIANG XH, XIE C, CHENG H, DA DONG J et al. Downregulation of CFTR promotes epithelial-to-mesenchymal transition and is associated with poor prognosis of breast cancer. Biochim Biophys Acta 2013; 1833: 2961-2969. https://doi.org/10.1016/j.bbamcr.2013.07.021

[20] NOMURA T, YAMASAKI M, NOMURA Y, MIMATA H. Expression of the inhibitors of apoptosis proteins in cisplatinresistant prostate cancer cells. Oncol Rep 2005; 14: 993-997. https://doi.org/10.3892/or.14.4.993

[21] GUMULEC J, BALVAN J, SZTALMACHOVA M, RAUDENSKA M, DVORAKOVA V et al. Cisplatin-resistant prostate cancer model: Differences in antioxidant system, apoptosis and cell cycle. Int J Oncol 2014; 44: 923-933. https://doi. org/10.3892/ijo.2013.2223

[22] YANG C, WU C, XU D, WANG M, XIA Q. AstragalosideII inhibits autophagic flux and enhance chemosensitivity of cisplatin in human cancer cells. Biomed Pharmacother 2016; 81: 166-175. https://doi.org/10.1016/j.biopha.2016.03.025

[23] KLIONSKY DJ. A human autophagy interaction network. Autophagy 2012; 8: 439-441. https://doi.org/10.4161/auto.19926

[24] FU WF, CHEN WB, DAI L, YANG GP, JIANG ZY et al. Inhibition of miR-141 reverses cisplatin resistance in non-small cell lung cancer cells via upregulation of programmed cell death protein 4. Eur Rev Med Pharmacol Sci 2016; 20: 2565-2572.

[25] KIM EM, MUELLER K, GARTNER E, BOERNER J. Dasatinib is synergistic with cetuximab and cisplatin in triple-negative breast cancer cells. J Surg Res 2013; 185: 231-239. https://doi. org/10.1016/j.jss.2013.06.041

[26] ZHANG N, YANG GQ, SHAO XM, WEI L. GAS5 modulated autophagy is a mechanism modulating cisplatin sensitivity in NSCLC cells. Eur Rev Med Pharmacol Sci 2016; 20: 2271-2277.

[27] YANG ZJ, CHEE CE, HUANG S, SINICROPE FA. The role of autophagy in cancer: therapeutic implications. Mol Cancer Ther 2011; 10: 1533-1541. https://doi.org/10.1158/1535-7163. MCT-11-0047

[28] WHITE E. Deconvoluting the context-dependent role for autophagy in cancer. Nat Rev Cancer 2012; 12: 401-410. https:// doi.org/10.1038/nrc3262

[29] JI C, ZHANG L, CHENG Y, PATEL R, WU H et al. Induction of autophagy contributes to crizotinib resistance in ALKpositive lung cancer. Cancer Biol Ther 2014; 15: 570-577. https://doi.org/10.4161/cbt.28162 
[30] HIPPERT MM, O'TOOLE PS, THORBURN A. Autophagy in cancer: good, bad, or both? Cancer Res 2006; 66: 9349-9351. https://doi.org/10.1158/0008-5472.CAN-06-1597

[31] TURCOTTE S, GIACCIA AJ. Targeting cancer cells through autophagy for anticancer therapy. Curr Opin Cell Biol 2010; 22: 246-251. https://doi.org/10.1016/j.ceb.2009.12.007

[32] GOODALL ML, WANG T, MARTIN KR, KORTUS MG, KAUFFMAN AL et al. Development of potent autophagy inhibitors that sensitize oncogenic BRAF V600E mutant melanoma tumor cells to vemurafenib. Autophagy 2014; 10 : 1120-1136. https://doi.org/10.4161/auto.28594

[33] QIAO S, TAO S, ROJO DE LA VEGA M, PARK SL, VONDERFECHT AA et al. The antimalarial amodiaquine causes autophagic-lysosomal and proliferative blockade sensitizing human melanoma cells to starvation- and chemotherapyinduced cell death. Autophagy 2013; 9: 2087-2102. https:// doi.org/10.4161/auto.26506

[34] CAREW JS, KELLY KR, NAWROCKI ST. Autophagy as a target for cancer therapy: new developments. Cancer Manag Res 2012; 4: 357-365. https://doi.org/10.2147/ CMAR.S26133

[35] HARHAJI-TRAJKOVIC L, VILIMANOVICH U, KRAVICSTEVOVIC T, BUMBASIREVIC V, TRAJKOVIC V. AMPK-mediated autophagy inhibits apoptosis in cisplatintreated tumour cells. J Cell Mol Med 2009; 13: 3644-3654. https://doi.org/10.1111/j.1582-4934.2009.00663.x 